\title{
POSITIVE DEFINITE KERNELS ON FREE PRODUCT SEMIGROUPS AND UNIVERSAL ALGEBRAS
}

\author{
GELU POPESCU
}

\begin{abstract}
A method to construct positive definite operator-valued kernels on free product semigroups amalgamated over the identity is presented. A complete description of the structure of positive definite Toeplitz kernels on free products of semigroups is given.

In particular, these results leed to the generalized disc algebra $\mathscr{A}_{n}\left(G^{+}\right), n=2,3, \ldots, \infty$, and the reduced $C^{*}$-algebra $C_{r}^{*}\left(*_{n} G^{+}\right)$, where $G^{+}$is the unital semigroup of nonnegative elements of an additive subgroup $G$ of real numbers. The completely bounded (resp. contractive) representations of $\mathscr{A}_{n}\left(G^{+}\right), n=2,3, \ldots, \infty$, on a Hilbert space are characterized when $G^{+}$is commensurable.

As consequences, we obtain von Neumann type inequalities, isometric (resp. unitary) dilations, for a large class of semigroups of operators on Hilbert spaces.
\end{abstract}

\section{Introduction and preliminaries}

Let $P$ be a unital semigroup with neutral element $e$ and let $B(\mathscr{H})$ be the algebra of all bounded operators on a Hilbert space $\mathscr{H}$. A hermitian operatorvalued kernel on $P$ is a map

$$
K: P \times P \rightarrow B(\mathscr{H})
$$

with the property that $K(\sigma, \omega)=K(\omega, \sigma)^{*}(\sigma, \omega \in P)$. If

$$
\sum_{i, j=1}^{k}\left\langle K\left(\sigma_{i}, \sigma_{j}\right) h_{j}, h_{i}\right\rangle \geq 0
$$

for any $k \in \mathrm{N}$, for any $h_{1}, \ldots, h_{k} \in \mathscr{H}$, and $\sigma_{1}, \ldots, \sigma_{k} \in P$, then $K$ is said to be positive definite. A kernel $K$ on $P$ is called Toeplitz if $K(e, e)=I_{\mathscr{H}}$ and $K(\alpha \sigma, \alpha \omega)=K(\sigma, \omega)$ for any $\alpha, \sigma, \omega \in P$.

Let $P_{i}(i \in I \subset\{1,2, \ldots\})$ be unital semigroups and let $P:=* P_{i}$ be the free product semigroup amalgamated over the identity. For any hermitian operator-valued kernels $K_{i}: P_{i} \times P_{i} \rightarrow B(\mathscr{H})(i \in I)$, we define a free product kernel $K:=* K_{i}$ on $P$ such that $K(\sigma, \omega)=K(\omega, \sigma)^{*}(\sigma, \omega \in P)$ and

Partially supported by NSF DMS-9531954.

Received November 14, 1996. 


$$
\left.K\right|_{P_{i} \times P_{i}}=K_{i} \quad \text { for any } i \in I .
$$

We show in Section 2 that if $K_{i}(i \in I)$ are positive definite Toeplitz kernels on semigroups, then the free product kernel $K$ has the same properties. This extends Bozejko's result concerning positive definite operator-valued functions on free products of groups [B].

In Section 3, we study the structure of positive definite Toeplitz kernels on semigroups, extending the Naimark dilation theorem $([\mathrm{N}],[\mathrm{SzF}])$ to our setting.

The results of these two sections are combined in order to obtain isometric (resp. unitary) representations for free products of semigroups. Certain consequences of these results are considered in the next sections.

Let $G$ be a discrete subgroup of the additive group of real numbers and $G^{+}$ be the unital semigroup of nonnegative elements of $G$. Let $\left\{V_{i}(s)\right\}_{s \in G^{+}} \subset B(\mathscr{K})(i=1,2, \ldots, n)$ be $n$ orthogonal semigroups of isometries, i.e.,

$$
V_{i}(0)=I_{\mathscr{K}}, \quad V_{i}(s+t)=V_{i}(s) V_{i}(t)
$$

for any $s, t \in G^{+}, i=1,2, \ldots, n$, and such that

$$
V_{1}(s) V_{1}(s)^{*}+\cdots+V_{n}(s) V_{n}(s)^{*} \leq I_{\mathscr{K}}
$$

for any $s \in G^{+} \backslash\{0\}$. Here, $n=1,2, \ldots, \infty$ and by abusing notations, when $n=\infty$ we meen a sequence of semigroups. We denote by $C^{*}\left(\left\{V_{i}(s)\right\}\right.$ the $C^{*}$ algebra generated by the isometries $V_{i}(s), i=1,2, \ldots, n, s \in G^{+}$. This $C^{*}$ algebra has been studied by many authors in various particular cases. Coburn ([C1], [C2]) studied the $C^{*}$-algebra generated by one isometry (this corresponds to the case $G^{+}=\mathrm{N}, n=1$ ) and Douglas [Do] proved that the $C^{*}$-algebra generated by one semigroup of isometries $\left\{V_{1}(s)\right\}_{s \in G^{+}}$is either a quotient of $C(\hat{G})$ or a generalized Toeplitz algebra. The case when $G^{+}=\mathrm{N}, n=2,3, \ldots, \infty$, was studied by Cuntz [Cu]. In [D1], Dihn considered the case when $G^{+}$is a countable dense subsemigroup of $[0, \infty)$ and $n=2,3, \ldots, \infty$.

The $C^{*}$-algebras $C^{*}\left(\left\{V_{i}(s)\right\}\right)$, with $G^{+}$dense, are special cases of a family of $C^{*}$-algebras arising naturally from the Arveson-Powers-Robinson index theory of semigroups of endomorphisms of type I factors ([Ar2], [D2]).

In Sections 4, 5, we study the closed nonselfadjoint algebra generated by $V_{i}(s), i=1,2, \ldots, n, s \in G^{+}, \quad$ where $G^{+} \quad$ is commensurable and $n=2,3, \ldots, \infty$.

Define the free product semigroup

$$
*_{n} G^{+}:=G_{1}^{+} * \cdots * G_{n}^{+},
$$

where $G_{i}^{+}$is an isomorphic copy of $G^{+}$for any $i=1,2, \ldots, n$. Let $\mathscr{A}_{n}\left(G^{+}\right)$be 
the closed nonselfadjoint algebra on $\ell^{2}\left(*_{n} G^{+}\right)$generated by the operators of left multiplication by elements of $*_{n} G^{+}$, and let $C_{r}^{*}\left(*_{n} G^{+}\right)$be the $C^{*}$-algebra generated by them. Notice that, in the particular case when $G^{+}=\mathbf{Z}^{+}=\{0,1,2, \ldots\}, \mathscr{A}_{n}\left(\mathbf{Z}^{+}\right)$is the noncommutative disc algebra $\mathscr{A}_{n}$ introduced in [Po1] (see also [Po2]), and $C_{r}^{*}\left(*_{n} \mathrm{Z}^{+}\right)=C^{*}\left(S_{1}, \ldots, S_{n}\right)$ is the extension through compacts of the Cuntz algebra $\mathcal{O}_{n}(n \geq 2)[\mathrm{Cu}]$.

We prove in Section 5 that $\mathscr{A}_{n}\left(G^{+}\right)$is the universal algebra generated by $n$ semigroups of contractions $\left\{T_{i}(s)\right\}_{s \in G^{+}} \subset B(\mathscr{H})(i=1,2, \ldots, n)$ such that

$$
T_{1}(s) T_{1}(s)^{*}+\cdots+T_{n}(s) T_{n}(s)^{*} \leq I_{\mathscr{H}}
$$

for any $s \in G^{+} \backslash\{0\}$.

It is clear that a sequence of semigroups of contractions $\mathscr{T}=\left\{T_{i}(s)\right\}_{s \in G^{+}} \subset B(\mathscr{H}), i=1,2, \ldots, n$, satisfying (1.1), gives rise to a contractive representation

$$
\rho_{\tau}: *_{n} G^{+} \rightarrow B(\mathscr{H})
$$

by setting

$$
\rho_{\tau}(g)=T(g)=T_{i_{1}}\left(t_{i_{1}}\right) \cdots T_{i_{k}}\left(t_{i_{k}}\right)
$$

if $g=t_{i_{1}} * t_{i_{2}} * \cdots * t_{i_{k}} \in *_{n} G^{+}$, with $i_{1} \neq i_{2} \neq \cdots \neq i_{k}, i_{1}, \ldots, i_{k} \in\{1,2, \ldots, n\}$, and $\rho_{\tau}(0)=I_{\mathscr{H}}$. Let $\mathscr{P}\left(*_{n} G^{+}\right)$be the set of formal linear combinations of elements of $*_{n} G^{+}$with the obvious algebra structure. An element $p \in \mathscr{P}\left(*_{n} G^{+}\right)$ has the form

$$
p=\sum_{\text {finite }} a_{g} g, \quad a_{g} \in \mathrm{C}, g \in *_{n} G^{+} .
$$

Define $\tilde{\rho}_{\tau}: \mathscr{P}\left(*_{n} G^{+}\right) \rightarrow B(\mathscr{H})$ by

$$
\tilde{\rho}_{\tau}(p)=\sum a_{g} \rho_{\tau}(g), \quad p=\sum_{\text {finite }} a_{g} g .
$$

We prove in Section 4 that if $G^{+}$is commensurable, then the contractive representation $\rho_{\tau}$ admits a minimal isometric dilation, i.e., there is a Hilbert space $\mathscr{K} \supset \mathscr{H}$ and an isometric representation $\pi: *_{n} G^{+} \rightarrow B(\mathscr{H})$ determined by semigroups of isometries $\left\{V_{i}(s)\right\}_{s \in G^{+}} \subset B(\mathscr{K}), i=1,2, \ldots, n$, satisfying the properties:

(i) $V_{1}(t) V_{1}(t)^{*}+\cdots+V_{n}(t) V_{n}(t)^{*} \leq I_{\mathscr{K}}$, for any $t \in G^{+} \backslash\{0\}$;

(ii) $\rho_{\tau}(g)=\left.P_{\mathscr{H}} \pi(g)\right|_{\mathscr{H}}, \quad g \in *_{n} G^{+}$;

(iii) $\mathscr{K}=\bigvee_{g \in *_{n} G^{+}} \pi(g) \mathscr{H}$.

Moreover, the minimal isometric dilation satisfying these properties is uniquely determined up to an isomorphism. Using the results from Sections 2,3 , we extend this result to a more general setting. 
In Section 5, we prove the following extension of the von Neumann inequality ([vN], [Po1])

$$
\left\|\tilde{\rho}_{\tau}(p)\right\| \leq\|p\|_{C_{r}^{*}\left(*_{n} G^{+}\right)} \leq\|p\|_{C^{*}\left(*_{n} G\right)}, \quad p \in \mathscr{P}\left(*_{n} G^{+}\right),
$$

where any polynomial $p$ is viewed as an element of the reduced $C^{*}$-algebra $C_{r}^{*}\left(*_{n} G^{+}\right)$(resp. the full group $C^{*}$-algebra $C^{*}\left(*_{n} G\right)$ ). Notice that in the particular case when $G^{+}=\mathrm{Z}^{+}$we find the noncommutative von Neumann inequality proved in [Po1] (see also [Po2], [Po4]). Moreover, the inequality (1.2) is extended to matrices.

Let us remark that all the results of this paper hold true for $n=\infty$ in a slightly adapted version.

In a subsequent paper we will study the generalized disc algebra $\mathscr{A}_{n}\left(G^{+}\right)$ in a more general setting.

\section{Positive definite kernels on free products of semigroups}

Let $P_{1}, P_{2}$ be unital semigroups and let $P:=P_{1} * P_{2}$ be the unital free product semigroup amalgamated over the identity $e$. We can assume that $P_{1} \cap P_{2}=\{e\}$. If $x \in P \backslash\{e\}$, then it has the unique representation

$$
x=\alpha_{1, i_{1}} \alpha_{2, i_{2}} \cdots \alpha_{k, i_{k}}
$$

where $\alpha_{j, i_{j}} \in P_{i_{j}} \backslash\{e\}, i_{j} \in\{1,2\}, i_{j} \neq i_{j+1}, j=1,2, \ldots, k-1$.

Let us introduce the block length function || on the free product semigroup $P$ setting $|e|=0$ and $|x|=k$ if $x \in P$ is of the form (2.1). For each $r=1,2, \ldots,|x|$, let $s_{r}(x)$ be the $r^{\text {th }}$ factor in the representation (2.1) of $x$. Notice that we have

$$
x=s_{1}(x) s_{2}(x) \cdots s_{|x|}(x) .
$$

Let $K_{i}: P_{i} \times P_{i} \rightarrow B(\mathscr{H}) \quad(i=1,2)$ be kernels such that $K_{i}\left(\omega_{i}, \sigma_{i}\right)=$ $K_{i}\left(\sigma_{i}, \omega_{i}\right)^{*}$ for any $\omega_{i}, \sigma_{i} \in P_{i}$. In the following we will define a kernel

$$
K: P \times P \rightarrow B(\mathscr{H})
$$

such that $K(\sigma, \omega)=K(\omega, \sigma)^{*}(\sigma, \omega \in P)$ and $\left.K\right|_{P_{i} \times P_{i}}:=K_{i} \quad(i=1,2)$. If $x \in P \backslash\{e\}$ has the representation (2.1) we define

$$
K(e, x):=K_{i_{1}}\left(e, \alpha_{1, i_{1}}\right) \cdots K_{i_{k}}\left(e, \alpha_{k, i_{k}}\right)
$$

and

$$
K(x, e):=K(e, x)^{*}=K_{i_{k}}\left(\alpha_{k, i_{k}}, e\right) \ldots K_{i_{1}}\left(\alpha_{1, i_{1}}, e\right) .
$$

Let $\omega, \sigma \in P \backslash\{e\}$ be such that $s_{1}(\omega) \neq s_{1}(\sigma)$. We have two subcases. If $\left\{s_{1}(\omega), s_{1}(\sigma)\right\} \subset P_{i}(i=1,2)$ we define 


$$
K(\omega, \sigma):=K\left(s_{2}(\omega) \cdots s_{|\omega|}(\omega), e\right) K_{i}\left(s_{1}(\omega), s_{1}(\sigma)\right) K\left(e, s_{2}(\sigma) \cdots s_{|\sigma|}(\sigma)\right) .
$$

If $\left\{s_{1}(\omega), s_{1}(\sigma)\right\} \not \subset P_{i}(i=1,2)$ then we set

$$
K(\omega, \sigma):=K(\omega, e) K(e, \sigma) .
$$

Now, let $\omega, \sigma \in P \backslash\{e\}$ be such that $s_{1}(\omega)=s_{1}(\sigma)$. Let $r$ be the maximum natural number such that $s_{i}(\omega)=s_{i}(\sigma)$ for any $i=1,2, \ldots, r$. Define

$$
K(\omega, \sigma):= \begin{cases}K\left(s_{r+1}(\omega) \cdots s_{|\omega|}(\omega), s_{r+1}(\sigma) \cdots s_{|\sigma|}(\sigma)\right) ; & \text { if } r<\min \{|\omega|,|\sigma|\} \\ K\left(e, s_{r+1}(\sigma) \cdots s_{|\sigma|}(\sigma)\right) ; & \text { if } r=|\omega|<|\sigma| \\ K\left(s_{r+1}(\omega) \cdots s_{|\omega|}(\omega), e\right) ; & \text { if } r=|\sigma|<|\omega| \\ I_{\mathscr{H}} ; & \text { if } r=|\omega|=|\sigma| .\end{cases}
$$

Since $\left.K\right|_{P_{i} \times P_{i}}=K_{i} \quad(i=1,2)$ and $K_{i}\left(\omega_{i}, \sigma_{i}\right)=K_{i}\left(\sigma_{i}, \omega_{i}\right)^{*}$ for any $\omega_{i}, \sigma_{i} \in P_{i}$, one can see that the kernel $K$, defined above, has the property $K(\omega, \sigma)=K(\sigma, \omega)^{*}$ for any $\omega, \sigma \in P$. Notice also that if $K_{i}(i=1,2)$ are Toeplitz kernels, then $K$ is also a Toeplitz kernel. The kernel $K$ is denoted by $K_{1} * K_{2}$ and called the free product of $K_{1}$ and $K_{2}$.

Let $S_{1}, S_{2}$ be two sets such that $S_{1} \cap S_{2}=\left\{x_{0}\right\}$, and let $K_{1}, K_{2}$ be kernels on $S_{1}, S_{2}$, respectively, such that $K_{1}\left(x_{0}, x_{0}\right)=K_{2}\left(x_{0}, x_{0}\right)=I$. Following [B], we define a kernel $K$ on $S_{1} \cup S_{2}$ in the following way:

(1) $\left.K\right|_{S_{i} \times S_{i}}=K_{i} \quad(i=1,2)$;

(2) (Markov property) For $s_{i} \in S_{i} \quad(i=1,2)$

$$
K\left(s_{1}, s_{2}\right):=K\left(s_{1}, x_{0}\right) K\left(x_{0}, s_{2}\right) \text { and } K\left(s_{1}, s_{2}\right)=K\left(s_{2}, s_{1}\right)^{*} .
$$

The kernel $K$ is denoted by $K_{1} *_{x_{0}}^{M} K_{2}$ and called the Markov product of $K_{1}$ and $K_{2}$.

Let us recall an important result of Bozejko [B] that will be used in what follows.

Lemma 2.1 (Bozejko). If $K_{1}, K_{2}$ are positive definite kernels on $S_{1}, S_{2}$, respectively, and $K_{i}(x, x)=I$ for all $x \in S_{1} \cup S_{2}$, then the Markov product $K=K_{1} *_{x_{0}}^{M} K_{2}$ is also a positive definite kernel.

Theorem 2.2. Let $P_{i}(i=1,2)$ be unital semigroups. If $K_{i}: P_{i} \times P_{i} \rightarrow$ $B(\mathscr{H})$ are positive definite kernels and $K_{i}\left(\sigma_{i}, \sigma_{i}\right)=I_{\mathscr{H}}$ for any $\sigma_{i} \in P_{i}$, then the free product $K_{1} * K_{2}$ is a positive definite kernel on $P_{1} * P_{2}$.

Proof. Since $P:=P_{1} * P_{2}$ is the free product semigroup amalgamated over the identity $e$, we can assume that $P_{1} \cap P_{2}=\{e\}$. Consider the following sets: $X_{0}=P_{1}, Y_{0}=P_{2}, \quad$ and for any $k=1,2, \ldots$, 


$$
\begin{aligned}
X_{2 k-1} & =\bigcup_{\substack{\alpha_{i} \in P_{1} \backslash\left\{\{\} \\
\beta_{i} \in P_{2} \backslash\{e\}\right.}}\left\{\alpha_{1} \beta_{1} \alpha_{2} \cdots \beta_{k-1} \alpha_{k} P_{2}\right\}, \\
X_{2 k} & =\bigcup_{\substack{\alpha_{i} \in P_{1} \backslash\left\{\{\} \\
\beta_{i} \in P_{2} \backslash\{e\}\right.}}\left\{\alpha_{1} \beta_{1} \alpha_{2} \cdots \alpha_{k} \beta_{k} P_{1}\right\} \\
Y_{2 k-1} & =\bigcup_{\substack{\alpha_{i} \in P_{1} \backslash\left\{\{\} \\
\beta_{i} \in P_{2} \backslash\{e\}\right.}}\left\{\beta_{1} \alpha_{1} \cdots \alpha_{k-1} \beta_{k} P_{1}\right\}, \\
Y_{2 k} & =\bigcup_{\substack{\alpha_{i} \in P_{1} \backslash\left\{\{\} \\
\beta_{i} \in P_{2} \backslash\{e\}\right.}}\left\{\beta_{1} \alpha_{1} \beta_{2} \cdots \beta_{k} \alpha_{k} P_{2}\right\} .
\end{aligned}
$$

Define also $X=\bigcup_{j=0}^{\infty} X_{j}$ and $Y=\bigcup_{j=0}^{\infty} Y_{j}$. Notice that $P_{1} * P_{2}=X \cup Y$ and $X \cap Y=\{e\}$. If $x \in X \backslash\{e\}$ and $y=Y \backslash\{e\}$ then $s_{1}(x) \neq s_{1}(y), s_{1}(x) \in P_{1}$ and $s_{1}(y) \in P_{2}$. According to the definition of $K:=K_{1} * K_{2}$, we infer that

$$
K(x, y)=K(x, e) K(e, y)=\left.\left.K\right|_{X \times X} *_{e}^{M} K\right|_{Y \times Y}(x, y) .
$$

Notice that this relation also holds if $x=e$ or $y=e$. If we can prove that the kernels $\left.K\right|_{X \times X}$ and $\left.K\right|_{Y \times Y}$ are positive definite, then, according to Lemma 2.1 , the kernel $K$ is positive definite.

Let us prove now that $\left.K\right|_{X \times X}$ is positive definite. For each $m=0,1,2, \ldots$ consider $Z_{m}=\bigcup_{j=0}^{m} X_{j}$. Since $X=\bigcup_{m=0}^{\infty} Z_{m}$ and $Z_{0} \subset Z_{1} \subset \cdots$ it is enough to prove that $\left.K\right|_{Z_{m} \times Z_{m}}$ is positive definite for any $m=0,1,2, \ldots$.

We proceed by induction. Notice that $\left.K\right|_{Z_{0} \times Z_{0}}=\left.K\right|_{P_{1} \times P_{1}}=K_{1}$ is positive definite. Assume that $\left.K\right|_{Z_{m} \times Z_{m}}$ is positive definite and let us prove that $\left.K\right|_{Z_{m+1} \times Z_{m+1}}$ is positive definite.

Suppose that $m=2 k-1$ is fixed (the proof is similar in the case when $m=2 k)$. Notice that $\left.K\right|_{Z_{2 k} \times Z_{2 k}}$ is positive definite if and only if $\left.K\right|_{\Lambda_{n} \times \Lambda_{n}}$ is positive definite for any $n=1,2, \ldots$, where

$$
\Lambda_{n}:=Z_{2 k-1} \bigcup\left(\bigcup_{r=1}^{n} \alpha_{1}^{(r)} \beta_{1}^{(r)} \cdots \alpha_{k}^{(r)} \beta_{k}^{(r)} P_{1}\right),
$$

and $\alpha_{i}^{(r)} \in P_{1} \backslash\{e\}, \beta_{i}^{(r)} \in P_{2} \backslash\{e\}, \quad i=1,2, \ldots, k, r=1,2, \ldots, n$, are fixed such that

$$
\alpha_{1}^{\left(r_{1}\right)} \beta_{1}^{\left(r_{1}\right)} \cdots \alpha_{k}^{\left(r_{1}\right)} \beta_{k}^{\left(r_{1}\right)} \neq \alpha_{1}^{\left(r_{2}\right)} \beta_{1}^{\left(r_{2}\right)} \cdots \alpha_{k}^{\left(r_{2}\right)} \beta_{k}^{\left(r_{2}\right)},
$$

if $r_{1}, r_{2} \in\{1,2, \ldots, n\}$ and $r_{1} \neq r_{2}$.

We proceed by induction over $j=0,1, \ldots, n$. Assume that $\left.K\right|_{\Lambda_{j} \times \Lambda_{j}}$ is positive definite for a fixed $j$ such that $0 \leq j \leq n-1$, where $\Lambda_{0}=Z_{2 k-1}$. We will 
show that $\left.K\right|_{\Lambda_{j+1} \times \Lambda_{j+1}}$ is positive definite. Notice that $\Lambda_{j} \cap\left\{y_{j+1} P_{1}\right\}=y_{j+1}$, where

$$
y_{j+1}=\alpha_{1}^{(j+1)} \beta_{1}^{(j+1)} \cdots \alpha_{k}^{(j+1)} \beta_{k}^{(j+1)},
$$

and $\Lambda_{j+1}=\Lambda_{j} \cup\left\{y_{j+1} P_{1}\right\}$. Since $K\left(y_{j+1} \omega, y_{j+1} \sigma\right)=K_{1}(\omega, \sigma)$ for any $\omega, \sigma \in P_{1}$, it follows that $\left.K\right|_{\left\{y_{j+1} P_{1}\right\} \times\left\{y_{j+1} P_{1}\right\}}$ is positive definite. Let us prove now that

$$
\left.K\right|_{\Lambda_{j+1} \times \Lambda_{j+1}}=\left.\left.K\right|_{\Lambda_{j} \times \Lambda_{j}} * *_{j+1}^{M} K\right|_{\left\{y_{j+1} P_{1}\right\} \times\left\{y_{j+1} P_{1}\right\}},
$$

that is,

$$
K\left(\lambda_{j}, y_{j+1} p_{1}\right)=K\left(\lambda_{j}, y_{j+1}\right) K\left(y_{j+1}, y_{j+1} p_{1}\right)
$$

for any $\lambda_{j} \in \Lambda_{j}$ and $p_{1} \in P_{1}$. We have two cases:

(i) $\lambda_{j} \in Z_{2 k-1}$;

(ii) $\lambda_{j}=y_{r} p_{1}^{\prime}$, where $y_{r}=\alpha_{1}^{(r)} \beta_{1}^{(r)} \cdots \alpha_{k}^{(r)} \beta_{k}^{(r)}, 1 \leq r \leq j$ and $p_{1}^{\prime} \in P_{1}$.

Case (i). We further divide the proof into two subcases:

(a) $s_{1}\left(\lambda_{j}\right) \neq s_{1}\left(y_{j+1} p_{1}\right)$;

(b) $s_{1}\left(\lambda_{j}\right)=s_{1}\left(y_{j+1} p_{1}\right)$.

Subcase (a). Since $\left\{s_{1}\left(\lambda_{j}\right), s_{1}\left(y_{j+1}\right)\right\} \subset P_{1} \backslash\{e\}$, according to the definition of $K$, we have

$$
\begin{aligned}
& K\left(\lambda_{j}, y_{j+1} p_{1}\right) \\
= & K\left(s_{2}\left(\lambda_{j}\right) \cdots s_{\left|\lambda_{j}\right|}\left(\lambda_{j}\right), e\right) K_{1}\left(s_{1}\left(\lambda_{j}\right), s_{1}\left(y_{j+1}\right)\right) K\left(e, s_{2}\left(y_{j+1}\right) \cdots s_{\left|y_{j+1}\right|}\left(y_{j+1}\right) p_{1}\right) \\
= & K\left(s_{2}\left(\lambda_{j}\right) \cdots s_{\left|\lambda_{j}\right|}\left(\lambda_{j}\right), e\right) K_{1}\left(s_{1}\left(\lambda_{j}\right), s_{1}\left(y_{j+1}\right)\right) K\left(e, s_{2}\left(y_{j+1}\right) \cdots\right. \\
& \left.\quad s_{\left|y_{j+1}\right|}\left(y_{j+1}\right)\right) K\left(e, p_{1}\right) \\
= & K\left(\lambda_{j}, y_{j+1}\right) K\left(e, p_{1}\right) \\
= & K\left(\lambda_{j}, y_{j+1}\right) K\left(y_{j+1}, y_{j+1} p_{1}\right) .
\end{aligned}
$$

Therefore, the relation (2.4) holds in this subcase.

Subcase (b). Notice that $\left|\lambda_{j}\right| \leq 2 k$ and $2 k \leq\left|y_{j+1} p_{1}\right| \leq 2 k+1$. Let $q$ be the maximum natural number such that $s_{i}\left(\lambda_{j}\right)=s_{i}\left(y_{j+1} p_{1}\right)$ for any $i=1,2, \ldots, q$. This shows that

$$
\lambda_{j}=s_{1}\left(\lambda_{j}\right) \cdots s_{q}\left(\lambda_{j}\right) \cdot s_{q+1}\left(\lambda_{j}\right) \cdots s_{\left|\lambda_{j}\right|}\left(\lambda_{j}\right)
$$

and

$$
y_{j+1} P_{1}=s_{1}\left(\lambda_{j}\right) \cdots s_{q}\left(\lambda_{j}\right) \cdot s_{q+1}\left(y_{j+1}\right) \cdots s_{\left|y_{j+1}\right|}\left(y_{j+1}\right) p_{1}
$$

with $\left\{s_{q+1}\left(\lambda_{j}\right), s_{q+1}\left(y_{j+1}\right)\right\} \subset P_{i}$ for some $i=1,2$, and $s_{q+1}\left(\lambda_{j}\right) \neq s_{q+1}\left(y_{j+1}\right)$. Using the definition of $K$, we have 


$$
K\left(\lambda_{j}, y_{j+1} p_{1}\right)=K\left(s_{q+1}\left(\lambda_{i}\right) \cdots s_{\left|\lambda_{j}\right|}\left(\lambda_{j}\right), s_{q+1}\left(y_{j+1}\right) \cdots s_{\left|y_{j+1}\right|}\left(y_{j+1}\right) p_{1}\right) .
$$

On the other hand, this is equal to

$$
\begin{aligned}
& K\left(s_{q+1}\left(\lambda_{j}\right) \cdots s_{\left|\lambda_{j}\right|}\left(\lambda_{j}\right), s_{q+1}\left(y_{j+1}\right) \cdots s_{\left|y_{j+1}\right|}\left(y_{j+1}\right)\right) K\left(e, p_{1}\right) \\
& =K\left(\lambda_{j}, y_{j+1}\right) K\left(y_{j+1}, y_{j+1} p_{1}\right),
\end{aligned}
$$

which proves that the relation (2.4) holds in subcase (b), therefore in case (i).

Case (ii). We divide the proof into two subcases:

( $\alpha) s_{1}\left(y_{r}\right) \neq s_{1}\left(y_{j+1}\right)$;

( $\beta) s_{1}\left(y_{r}\right)=s_{1}\left(y_{j+1}\right)$.

Subcase $(\alpha)$. Since $\left\{s_{1}\left(y_{r}\right), s_{1}\left(y_{j+1}\right)\right\} \subset P_{1}$ and $\left|y_{r}\right|=\left|y_{j+1}\right|=2 k$, we have

$$
\begin{aligned}
& K\left(\lambda_{j}, y_{j+1} p_{1}\right)=K\left(y_{r} p_{1}^{\prime}, y_{j+1} p_{1}\right) \\
& =K\left(s_{2}\left(y_{r}\right) \cdots s_{2 k}\left(y_{r}\right) p_{1}^{\prime}, e\right) K_{1}\left(s_{1}\left(y_{r}\right), s_{1}\left(y_{j+1}\right)\right) K\left(e, s_{2}\left(y_{j+1}\right) \cdots s_{2 k}\left(y_{j+1}\right)\right) K\left(e, p_{1}\right) \\
& =K\left(y_{r} p_{1}^{\prime}, y_{j+1}\right) K\left(e, p_{1}\right) \\
& =K\left(\lambda_{i}, y_{j+1}\right) K\left(y_{j+1}, y_{j+1} p_{1}\right) .
\end{aligned}
$$

Therefore, the relation (2.4) is satisfied.

Subcase $(\beta)$. Since $y_{r} \neq y_{j+1}$, there exists $1 \leq q \leq 2 k-1$ such that $s_{i}\left(y_{r}\right)=s_{i}\left(y_{j+1}\right)$ for any $i=1,2, \ldots, q$, and $s_{q+1}\left(y_{r}\right) \neq s_{q+1}\left(y_{j+1}\right)$. Assume that $q \leq 2 k-2$. Since $\left\{s_{q+1}\left(y_{r}\right), s_{q+1}\left(y_{j+1}\right)\right\} \subset P_{1}$ (resp. $P_{2}$ ) if $q$ is even (resp. $q$ is odd) we have

$$
\begin{aligned}
& K\left(\lambda_{j}, y_{j+1} p_{1}\right)=K\left(y_{r} p_{1}^{\prime}, y_{j+1} p_{1}\right) \\
& =K\left(s_{q+1}\left(y_{r}\right) \cdots s_{2 k}\left(y_{r}\right) p_{1}^{\prime}, s_{q+1}\left(y_{j+1}\right) \cdots s_{2 k}\left(y_{j+1}\right) p_{1}\right) \\
& =K\left(s_{q+2}\left(y_{r}\right) \cdots s_{2 k}\left(y_{r}\right) p_{1}^{\prime}, e\right) K\left(s_{q+1}\left(y_{r}\right), s_{q+1}\left(y_{j+1}\right)\right) K\left(e, s_{q+2}\left(y_{j+1}\right) \cdots\right. \\
& \left.\quad s_{2 k}\left(y_{j+1}\right) p_{1}\right) \\
& =K\left(y_{r} p_{1}^{\prime}, y_{j+1}\right) K\left(e, p_{1}\right)=K\left(y_{r} p_{1}^{\prime}, y_{j+1}\right) K\left(y_{j+1}, y_{j+1} p_{1}\right) \\
& =K\left(\lambda_{j}, y_{j+1}\right) K\left(y_{j+1}, y_{j+1} p_{1}\right),
\end{aligned}
$$

proving that the relation (2.4) holds. The case $q=2 k-1$ can be treated similarly. This completes the proof of subcase $(\beta)$ and also case (ii). Summing up the above results, we conclude that the relation (2.3) is true. Since the kernels $\left.K\right|_{\Lambda_{j} \times \Lambda_{j}}$ and $\left.K\right|_{\left\{y_{j+1} P_{1}\right\} \times\left\{y_{j+1} P_{1}\right\}}$ are positive, using Lemma 2.1, we infer that $\left.K\right|_{\Lambda_{j+1} \times \Lambda_{j+1}}$ is positive definite. The induction argument shows that $\left.K\right|_{\Lambda_{n} \times \Lambda_{n}}$ is positive definite for any $n=1,2, \ldots$. This shows that $\left.K\right|_{Z_{2 k} \times Z_{2 k}}$ is positive definite and completes our inductive argument to prove that $\left.K\right|_{Z_{m} \times Z_{m}}$ is positive definite for any $m=0,1,2, \ldots$. According to the remarks 
considered at the beginning of this proof, we infer that $\left.K\right|_{X \times X}$ is positive definite.

Similarly, one can prove that $\left.K\right|_{Y \times Y}$ is positive definite. Since

$$
K=\left.\left.K\right|_{X \times X} *_{e}^{M} K\right|_{Y \times Y},
$$

using Lemma 2.1 again, we deduce that $K$ is positive definite on $P_{1} * P_{2}$. This completes the proof.

Corollary 2.3. Let $P_{i}(i=1,2)$ be unital semigroups. If $K_{i}: P_{i} \times P_{i} \rightarrow$ $B(\mathscr{H}), K_{i}(e, e)=I_{\mathscr{H}}$, are positive definite Toeplitz kernels, then the free product $K_{1} * K_{2}$ is a positive definite Toeplitz kernel on $P_{1} * P_{2}$.

Let $G_{i}, i \in I \subset\{1,2, \ldots\}$, be groups and $G=*_{i \in I} G_{i}$ be the free product group. Consider $u_{i}: G_{i} \rightarrow B(\mathscr{H})$ such that $u_{i}(e)=I_{\mathscr{H}}$. We define the free product function $u:=*_{i \in I} u_{i}$ in the following way. If $x \in G, x \neq e$ and $x$ has the unique representation

$$
x=a_{1, i_{1}} \cdots a_{k, i_{k}},
$$

where $a_{j, i_{j}} \in G_{i_{j}}$ and $i_{j} \neq i_{j+1}, j=1, \ldots, k-1$, then we set

$$
u(x)=\prod_{i=1}^{k} u_{i_{j}}\left(a_{j, i_{j}}\right)
$$

and $u(e)=I_{\mathscr{H}}$. Let us recall that a function $f: G \rightarrow B(\mathscr{H})$ is positive definite if the kernel $K_{f}(y, x)=f\left(y^{-1} x\right)$ is positive definite in the sense of Section 1.

Corollary 2.4 (Bozejko). Let $G_{i}(i \in I)$ be groups. If $u_{i}: G_{i} \rightarrow B(\mathscr{H})$, $u_{i}(e)=I_{\mathscr{H}}$, are positive definite functions, then the free product function $u=*_{i \in I} u_{i}$ is positive definite on the free product group $G=*_{i \in I} G_{i}$.

Proof. According to Corollary 2.3, it is enough to prove that the kernel $K_{u}$ is equal to the free product kernel of $K_{u_{i}}(i \in I)$, i.e., $K_{u}=* K_{u_{i}}$ in the sense of the definition given in the beginning of this section. This is a straightforward computation, so we omit it.

Let us consider some examples of positive definite kernels on semigroups.

ExAmple 2.5. Let $N=\{1,2, \ldots\}$ be the additive semigroup of natural numbers and $T \in B(\mathscr{H})$. Define the kernel $K_{T}: \mathrm{N} \times \mathrm{N} \rightarrow B(\mathscr{H})$ by

$$
K_{T}(n, m)= \begin{cases}T^{m-n} ; & \text { if } m>n \\ T^{* n-m} ; & \text { if } m<n \\ I_{\mathscr{H}} ; & \text { if } m=n\end{cases}
$$


It is well-known that $K_{T}$ is positive definite if and only if $T$ is a contraction (see $[\mathrm{SzF}]$ ).

EXAMPLe 2.6. Let $\mathrm{R}^{+}$be the additive unital semigroup of positive real numbers and let $\tau=\{T(t)\}_{t>0} \subset B(\mathscr{H})$ be a strongly continuous semigroup of contractions. Define $K_{\tau}: \mathrm{R}^{+} \times \mathrm{R}^{+} \rightarrow B(\mathscr{H})$ by

$$
K_{\tau}(t, s)= \begin{cases}T(s-t) ; & \text { if } s>t \\ T(t-s)^{*} ; & \text { if } s<t \\ I_{\mathscr{H}} ; & \text { if } s=t .\end{cases}
$$

The Toeplitz kernel $K_{\tau}$ is positive definite [SzF].

EXAmple 2.7. Let $\mathrm{F}_{\mathrm{n}}^{+}$be the unital free semigroup on $n$ generators: $s_{1}, \ldots, s_{n}$. Consider $T_{1}, \ldots, T_{n} \in B(\mathscr{H})$ and set $T(\omega):=T_{i_{1}} \cdots T_{i_{k}}$ for any $\omega=s_{i_{1}} \cdots s_{i_{k}}$, and $T(e)=I_{\mathscr{H}}$. Let $K_{\left[T_{1}, \ldots, T_{n}\right]}: \mathrm{F}_{n}^{+} \times \mathrm{F}_{n}^{+} \rightarrow B(\mathscr{H})$ be the Toeplitz kernel defined by

$$
K_{\left[T_{1}, \ldots, T_{n}\right]}(\sigma, \omega)= \begin{cases}T(\delta) ; & \text { if } \omega=\sigma \delta \text { for some } \delta \in \mathrm{F}_{n}^{+} \\ T(\delta)^{*} ; & \text { if } \sigma=\omega \delta \text { for some } \delta \in \mathrm{F}_{n}^{+} \\ 0 ; & \text { otherwise. }\end{cases}
$$

It was proved in [Po3] that $K_{\left[T_{1}, \ldots, T_{n}\right]}$ is positive definite if and only if the operator matrix $\left[T_{1}, \ldots, T_{n}\right]$ is a contraction. Let us remark that

$$
K_{\left[T_{1}, \ldots, T_{n}\right]} \neq K_{T_{1}} * \cdots * K_{T_{n}} .
$$

The following example shows that one can define many different positive definite Toeplitz kernels on the unital free semigroup on $n$ generators.

EXAMPLe 2.8. Let $n, n_{1}, n_{2}, \ldots, n_{k} \in\{1,2, \ldots\}$ such that $n=n_{1}+n_{2}+\ldots+$ $n_{k}$, and consider $\left\{T_{i j}\right\}_{\substack{i=1,2, \ldots, k \\ j=1,2, n_{i}}} \subset B(\mathscr{H})$ satisfying the relation

$$
T_{i 1} T_{i 1}^{*}+\cdots+T_{i n_{i}} T_{i n_{i}}^{*} \leq I_{\mathscr{H}}, \quad i=1,2, \ldots, k .
$$

According to Corollary 2.3, the Toeplitz kernel

$$
K=K_{\left[T_{11}, \ldots, T_{1 n_{1}}\right]} * \cdots * K_{\left[T_{k 1}, \ldots, T_{k n_{k}}\right]}
$$

is positive definite on $\mathrm{F}_{n}^{+}$.

Other examples of positive definite Toeplitz kernels on free semigroups are considered in [Po3], [Po4], and also in Section 4.

\section{The structure of positive definite Toeplitz kernels}

Let $P$ be a unital semigroup and let $K: P \times P \rightarrow B(\mathscr{H})$ be an operatorvalued kernel such that $K(e, e)=I_{\mathscr{H}}$. An isometric representation of $P$ on a 
Hilbert space $\mathscr{K}$ is a function $V: P \rightarrow B(\mathscr{K})$, where values are isometric operators on $\mathscr{K}$, and such that $V(e)=I_{\mathscr{K}}, V(x y)=V(x) V(y)$ for any $x, y \in P$. We will denote such a semigroup of isometries by $\{V(x)\}_{x \in P}$. We say that $K$ has a Naimark dilation if there is a Hilbert space $\mathscr{K} \supset \mathscr{H}$ and $\{V(\sigma)\}_{\sigma \in P}$ a semigroup of isometries on $\mathscr{K}$ such that

$$
K(\sigma, \omega)=\left.P_{\mathscr{H}} V(\sigma)^{*} V(\omega)\right|_{\mathscr{H}} \quad \text { for any } \sigma, \omega \in P,
$$

where $P_{\mathscr{H}}$ is the orthogonal projection of $\mathscr{K}$ onto $\mathscr{H}$. The Naimark dilation is called minimal if $\mathscr{K}=\bigvee_{\sigma \in P} V(\sigma) \mathscr{H}$.

Definition 3.1. Let $K: P \times P \rightarrow B(\mathscr{H})$ be an operator-valued kernel. We say that

(i) $K$ is $\tau$-Toeplitz $(\tau \in P)$ if

$$
K(\tau \sigma, \tau \omega)=K(\sigma, \omega)
$$

for any $\sigma, \omega \in P$;

(ii) $K$ is Toeplitz if it is $\tau$-Toeplitz for any $\tau \in P$;

(iii) $K$ is $\tau$-bounded $(\tau \in P)$ if there exists $M_{\tau}>0$ such that

$$
\sum_{\sigma, \omega \in P}\left\langle\left\langle K(\tau \sigma, \tau \omega) h_{\omega}, h_{\sigma}\right\rangle \leq M_{\tau} \sum_{\sigma, \omega \in P}\left\langle\left\langle K(\sigma, \omega) h_{\omega}, h_{\sigma}\right\rangle\right.\right.
$$

for any finitely supported sequence $\left\{h_{\sigma}\right\}_{\sigma \in P}$ in $\mathscr{H}$;

(iv) $K$ is bounded if it is $\tau$-bounded for any $\tau \in P$;

(v) $K$ is uniformly bounded if it is bounded and

$$
\sup _{\tau \in P} M_{\tau}<\infty \text {. }
$$

Notice that if $K$ is Toeplitz, then it is also uniformly bounded.

In what follows we extend the Naimark dilation theorem to our setting. The proof is modeled on the ideas of the classical result [N] and also [Po3].

TheOrem 3.2. Let $P$ be a unital semigroup and let $K: P \times P \rightarrow B(\mathscr{H})$ be an operator-valued kernel such that $K(e, e)=I_{\mathscr{H}}$. Then $K$ is a positive definite Toeplitz kernel if and only if it admits a minimal Naimark dilation, that is, there is a Hilbert space $\mathscr{K} \supset \mathscr{H}$ and $\{V(\sigma)\}_{\sigma \in P}$ a semigroup of isometries on $\mathscr{K}$ such that

$$
K(\sigma, \omega)=\left.P_{\mathscr{H}} V(\sigma)^{*} V(\omega)\right|_{\mathscr{H}} \quad \text { for any } \sigma, \omega \in P,
$$

and $\mathscr{K}=\bigvee_{\sigma \in P} V(\sigma) \mathscr{H}$. In this case its minimal Naimark dilation is unique up to an isomorphism.

Moreover, if $P$ is a topological semigroup and $K(\sigma, \omega)$ is weakly continuous 
with respect to each variable $\sigma, \omega \in P$, then $\{V(\sigma)\}_{\sigma \in P}$ is also a weakly continuous function of $\sigma$.

Proof. Assume $K: P \times P \rightarrow B(\mathscr{H})$ is a positive definite Toeplitz kernel. Let $\mathscr{K}_{0}$ be the set of all finitely supported sequences $\left\{h_{\sigma}\right\}_{\sigma \in P}$ in $\mathscr{H}$. Define the bilinear form $\langle\cdot, \cdot\rangle$ on $\mathscr{K}_{0}$ by

$$
\left\langle\left\{h_{\omega}\right\}_{\omega \in P},\left\{k_{\sigma}\right\}_{\sigma \in P}\right\rangle:=\sum_{\omega, \sigma \in P}\left\langle K(\sigma, \omega) h_{\omega}, k_{\sigma}\right\rangle_{\mathscr{H}} .
$$

Since $K$ is positive definite, $\langle\cdot, \cdot\rangle$ is positive semi-definite. Consider

$$
\mathscr{N}=\left\{k \in \mathscr{K}_{0}:\langle k, k\rangle=0\right\}
$$

and the quotient space $\mathscr{K}_{0 / \mathcal{N}}$. Let $\mathscr{K}$ be the Hilbert space obtained by completing $\mathscr{K}_{0 / \mathcal{N}}$ with the induced inner product. For each $\tau \in P$ let us define the operator $V(\tau)$ on $\mathscr{K}_{0}$ by

$$
V(\tau)\left\{h_{\sigma}\right\}_{\sigma \in P}=\left\{\sum_{\sigma \in P} \delta_{\tau \sigma}(t) h_{\sigma}\right\}_{t \in P},
$$

where $\delta_{\tau \sigma}(t)=1$ if $t=\tau \sigma$ and $\delta_{\tau \sigma}(t)=0$ otherwise. It is easy to see that $V(\tau)$ is well-defined on $\mathscr{K}_{0}, V(e)=I_{\mathscr{K}_{0}}$, and $V(\omega) V(\tau)=V(\omega \tau)$ for any $\omega, \tau \in P$. Let us show that $V(\tau)$ is an isometry on $\mathscr{K}_{0}$. Indeed, since $K$ is a Toeplitz kernel, i.e., $K(\tau \sigma, \tau \omega)=K(\sigma, \omega)$ for any $\tau, \sigma, \omega \in P$, we have

$$
\begin{aligned}
(3.2)\left\langle V(\tau)\left(\left\{h_{\omega}\right\}\right), V(\tau)\left(\left\{h_{\sigma}^{\prime}\right\}\right)\right\rangle & =\sum_{s, t \in P}\left\langle K(s, t) \sum_{\omega \in P} \delta_{\tau \omega}(t) h_{\omega}, \sum_{\sigma \in P} \delta_{\tau \sigma}(s) h_{\sigma}^{\prime}\right\rangle \\
& =\sum_{\sigma, \omega \in P}\left\langle K(\tau \sigma, \tau \omega) h_{\omega}, h_{\sigma}^{\prime}\right\rangle \stackrel{(*)}{=} \sum_{\sigma, \omega \in P}\left\langle K(\sigma, \omega) h_{\omega}, h_{\sigma}^{\prime}\right\rangle \\
& =\left\langle\left\{h_{\omega}\right\},\left\{h_{\sigma}^{\prime}\right\}\right\rangle .
\end{aligned}
$$

Therefore, the operator $V(\tau)$ extends by continuity to an isometry on $\mathscr{K}$, denoted also by $V(\tau)$, and $\{V(\tau)\}_{\tau \in P}$ is an isometric representation of $P$. Embed $\mathscr{H}$ in $\mathscr{K}$ by setting $\left.h=\left\{\delta_{e}(t) h\right)\right\}_{t \in P}$ where

$$
\delta_{e}(t)= \begin{cases}1 ; & \text { if } t=e \\ 0 ; & \text { if } t \neq e .\end{cases}
$$

This identification is allowed since it preserves the linear and metric structure of $\mathscr{H}$. Indeed we have

$$
\begin{aligned}
\left\langle\delta_{e}(s) h, \delta_{e}(t) h^{\prime}\right\rangle_{\mathscr{K}} & =\sum_{t, s \in P}\left\langle K(t, s) \delta_{e}(s) h, \delta_{e}(t) h^{\prime}\right\rangle_{\mathscr{H}} \\
& =\left\langle K(e, e) h, h^{\prime}\right\rangle_{\mathscr{H}}=\left\langle h, h^{\prime}\right\rangle_{\mathscr{H}} .
\end{aligned}
$$


For any $h, h^{\prime} \in \mathscr{H}$ and $\sigma, \omega \in P$ we have

$$
\begin{aligned}
\left\langle V(\omega)^{*} V(\sigma) h, h^{\prime}\right\rangle_{\mathscr{K}} & =\left\langle\left\{\delta_{\sigma}(t) h\right\},\left\{\delta_{\omega}(t) h^{\prime}\right\}\right\rangle_{\mathscr{K}} \\
& =\sum_{t, s \in P}\left\langle K(t, s) \delta_{\sigma}(s) h, \delta_{\omega}(t) h^{\prime}\right\rangle_{\mathscr{H}} \\
& =\left\langle K(\omega, \sigma) h, h^{\prime}\right\rangle_{\mathscr{H}},
\end{aligned}
$$

which implies $\left.P_{\mathscr{H}} V(\omega)^{*} V(\sigma)\right|_{\mathscr{H}}=K(\omega, \sigma)$ for any $\omega, \sigma \in P$.

Let us observe that every element in $\mathscr{K}_{0}$ can be considered as a finite sum of terms of type $\left\{\delta_{\sigma}(t) h\right\}_{t \in P}(h \in \mathscr{H})$ and hence every element $k \in \mathscr{K}_{0}$ can be decomposed into a finite sum of terms of the type $V(\sigma) h, \sigma \in P, h \in \mathscr{H}$. This implies $\mathscr{K}=\bigvee_{\sigma \in P} V(\sigma) \mathscr{H}$, i.e., the Naimark dilation is minimal.

To prove the uniqueness, let $\left\{V^{\prime}(s)\right\}_{s \in P}$ be another minimal dilation of $K$ on a Hilbert space $\mathscr{K}^{\prime} \supset \mathscr{H}$. One can prove that there is a unitary operator $W: \mathscr{K} \rightarrow \mathscr{K}^{\prime}$ such that $W V(\omega)=V^{\prime}(\omega) W$ for any $\omega \in P$, and $\left.W\right|_{\mathscr{H}}=I_{\mathscr{H}}$. To see this, it is sufficient to define

$$
W\left(\sum_{\sigma \in P}^{\text {finite }} V(\sigma) h_{\sigma}\right)=\sum_{\sigma \in P}^{\text {finite }} V^{\prime}(\sigma) h_{\sigma} \quad\left(h_{\sigma} \in \mathscr{H}\right) .
$$

Since $\left\langle V(\sigma) h, V(\omega) h^{\prime}\right\rangle_{\mathscr{K}}=\left\langle K(\omega, \sigma) h, h^{\prime}\right\rangle_{\mathscr{H}}$ for any $h, h^{\prime} \in \mathscr{H}$ and $\sigma, \omega \in P$, one can easily see that the operator $W$ defined by (3.3) is correctly defined, isometric, and in view of minimality, it extends to a unitary operator between $\mathscr{K}$ and $\mathscr{K}^{\prime}$.

To prove the converse of this theorem, let $\{V(\sigma)\}_{\sigma \in P}$ be an isometric representation of $P$ on a Hilbert space $\mathscr{K} \supset \mathscr{H}$. Assume that $K: P \times P \rightarrow$ $B(\mathscr{H})$ is the kernel defined by

$$
K(\omega, \sigma)=\left.P_{\mathscr{H}} V(\omega)^{*} V(\sigma)\right|_{\mathscr{H}}, \quad \text { for any } \omega, \sigma \in P .
$$

It is easy to see that $K(e, e)=I_{\mathscr{H}}, K(\omega, \sigma)=K(\sigma, \omega)^{*}$ and $K(s \omega, s \sigma)=$ $K(\omega, \sigma)$ for any $s, \omega, \sigma \in P$. Since for any finitely supported sequence $\left\{h_{\omega}\right\}_{\omega \in P} \subset \mathscr{H}$

$$
\begin{aligned}
\sum_{\sigma, \omega \in P}\left\langle K(\sigma, \omega) h_{\omega}, h_{\sigma}\right\rangle & =\sum_{\sigma, \omega \in P}\left\langle P_{\mathscr{H}} V(\sigma)^{*} V(\omega) h_{\omega}, h_{\sigma}\right\rangle \\
& =\left\|\sum_{\omega \in P} V(\omega) h_{\omega}\right\|^{2} \geq 0
\end{aligned}
$$

we infer that $K$ is a positive definite Toeplitz kernel.

It remains to consider the case when $P$ is a topological semigroup and $K(\sigma, \omega)$ is weakly continuous with respect to each variable $\sigma, \omega \in P$. Since $\|V(\sigma)\|=1$ for any $\sigma \in P$ and $\mathscr{K}=\bigvee_{\sigma \in P} V(\sigma) \mathscr{H}$, it is enough to prove that 
$\left\langle V(s) V(\sigma) h, V(\tau) h^{\prime}\right\rangle$ is a continuous function of $s$ for any $h, h^{\prime} \in \mathscr{H}$ and $\sigma, \omega \in P$. Notice that

$$
\left\langle V(s) V(\sigma) h, V(\tau) h^{\prime}\right\rangle_{\mathscr{K}}=\left\langle P_{\mathscr{H}} V(\tau)^{*} V(s \sigma) h, h^{\prime}\right\rangle=\left\langle K(\tau, s \sigma) h, h^{\prime}\right\rangle
$$

and this is a continuous function of $s$. This completes the proof.

A closer look at (3.2) reveals that $V(\tau)$ is an isometry due to the only fact that $K$ is $\tau$-Toeplitz. If we drop out the condition $\left(\mathrm{T}_{\tau}\right)$, then $V(\tau)$ is a bounded operator on $\mathscr{K}_{0}$ if and only if $K$ is $\tau$-bounded. Notice that if we replace the equality $(*)$ from $(3.2)$ by the inequality $\left(\mathrm{M}_{\tau}\right)$ the proof of Theorem 3.2 still works. Consequently, if $K$ is a positive definite bounded kernel in Theorem 3.2, then $\{V(\sigma)\}_{\sigma \in P}$ is a semigroup of bounded operators. The last part of the theorem remains true if we assume that $K$ is uniformly bounded.

Remark 3.3. Let $P$ be a unital semigroup and let $K: P \times P \rightarrow B(\mathscr{H})$ be a positive definite kernel with $K(e, e)=I_{\mathscr{H}}$. Let $\{V(\sigma)\}_{\sigma \in P} \subset B\left(\mathscr{K}_{0}\right)$ be the associated semigroup of operators, as in the proof of Theorem 3.2. Then the following statements are true:

(i) $V(\tau)$ is bounded if and only if $K$ is $\tau$-bounded;

(ii) $V(\tau)$ is an isometry if and only if $K$ is $\tau$-Toeplitz;

(iii) $V(\tau) \mathscr{K}_{0} \perp V(s) \mathscr{K}_{0}(\tau \neq s)$ if and only if $K(\tau \sigma, s \omega)=0$ for any $\sigma, \omega \in P$;

(iv) If $K$ is $\tau$-Toeplitz and $\tau P=P$, then $V(\tau)$ is unitary;

(v) If $P$ is a $*$-semigroup and $K$ is $\tau$-bounded, then $V\left(\tau^{*}\right)=V(\tau)^{*}$ if and only if

$$
K(\sigma, \tau \omega)=K\left(\tau^{*} \sigma, \omega\right)
$$

for any $\sigma, \omega \in P$. Moreover, if (3.4) holds and $\tau^{*} \tau=e$, then $K$ is $\tau$-Toeplitz, so $V(\tau)$ is an isometry.

COROLlaRy 3.4 (Naimark). If $G$ is a group and $f: G \rightarrow B(\mathscr{H})$ is a positive definite function with $f(e)=I_{\mathscr{H}}$, then there exists a Hilbert space $\mathscr{K} \supset \mathscr{H}$ and a unitary representation $U: G \rightarrow B(\mathscr{K})$ such that

$$
f(\sigma)=\left.P_{\mathscr{H}} U(\sigma)\right|_{\mathscr{H}}, \quad \sigma \in G,
$$

and $\mathscr{K}=\bigvee_{\sigma \in G} U(\sigma) \mathscr{H}$.

Proof. Let $P=G$ and let $K: G \times G \rightarrow B(\mathscr{H})$ be defined by $K(\sigma, \omega)=f\left(\sigma^{-1} \omega\right), \sigma, \omega \in G$. Since $K$ is a positive definite Toeplitz kernel on $G$, the result follows from Theorem 3.2 and Remark 3.3 part (iv).

Theorem 3.5. Let $P_{1}, \ldots, P_{n}$ be unital semigroups and let $K_{i}: P_{i} \times P_{i} \rightarrow$ 
$B(\mathscr{H})(i=1,2, \ldots, n)$ be positive definite Toeplitz kernels. Then there exists a Hilbert space $\mathscr{K} \supset \mathscr{H}$ and a semigroup $\{V(\sigma)\}_{\sigma \in P_{1} * \cdots * P_{n}}$ of isometries on $\mathscr{K}$ such that

$$
\left(K_{1} * \cdots * K_{n}\right)(\sigma, \omega)=\left.P_{\mathscr{H}} V(\sigma)^{*} V(\omega)\right|_{\mathscr{H}}
$$

for any $\sigma, \omega \in P_{1} * \cdots * P_{n}$, and $\mathscr{K}=\bigvee_{\sigma \in P_{1} * \cdots * P_{n}} V(\sigma) \mathscr{H}$.

Proof. According to Corollary 2.3, the free product kernel $K_{1} * \cdots * K_{n}$ is a positive definite Toeplitz kernel on the free product semigroup $P_{1} * \cdots * P_{n}$. Now, we can use Theorem 3.2 to complete the proof.

Let $P$ be a unital semigroup and let $\{T(\sigma)\}_{\sigma \in P} \subset B(\mathscr{H})$ be a semigroup of contractions, i.e.,

$$
T(0)=I_{\mathscr{H}}, T(\sigma \omega)=T(\sigma) T(\omega)
$$

for any $\sigma, \omega \in P$. We say that $\{T(\sigma)\}_{\sigma \in P}$ has a minimal isometric dilation if there is a Hilbert space $\mathscr{K} \supset \mathscr{H}$ and a semigroup $\{V(\sigma)\}_{\sigma \in P} \subset B(\mathscr{K})$ of isometries such that

$$
T(\sigma)=\left.P_{\mathscr{H}} V(\sigma)\right|_{\mathscr{H}}, \quad \sigma \in P,
$$

and $\mathscr{K}=\bigvee_{\sigma \in P} V(\sigma) \mathscr{H}$.

Corollary 3.6. Let $P_{i}(i=1,2, \ldots, n)$ be unital semigroups and let $\{T(\sigma)\}_{\sigma \in P_{i}} \subset B(\mathscr{H})(i=1,2, \ldots, n)$ be semigroups of contractions that have isometric dilations. Then there is a Hilbert space $\mathscr{K} \supset \mathscr{H}$ and $\{V(\sigma)\}_{\sigma \in P_{1} * \cdots * P_{n}} \subset B(\mathscr{K})$, an isometric representation of $P_{1} * \cdots * P_{n}$, such that

$$
T\left(\sigma_{1}\right) \cdots T\left(\sigma_{k}\right)=\left.P_{\mathscr{H}} V\left(\sigma_{1}\right) \cdots V\left(\sigma_{k}\right)\right|_{\mathscr{H}}
$$

for any $\sigma_{1}, \ldots, \sigma_{k} \in \cup_{i=1}^{n} P_{i}$, and $\mathscr{K}=\bigvee_{\sigma \in P_{1} * \cdots * P_{n}} V(\sigma) \mathscr{H}$.

Proof. For each $i=1,2, \ldots, n$, let $\{W(\sigma)\}_{\sigma \in P_{i}} \subset B\left(\mathscr{K}_{i}\right)$ be an isometric dilation of $\{T(\sigma)\}_{\sigma \in P_{i}}$ on a Hilbert Space $\mathscr{K}_{i} \supset \mathscr{H}$. Define the kernel $K_{i}: P_{i} \times P_{i} \rightarrow B(\mathscr{H})$ by

$$
K_{i}(\omega, \sigma)=\left.P_{\mathscr{H}} W(\omega)^{*} W(\sigma)\right|_{\mathscr{H}}
$$

for any $\omega, \sigma \in P_{i}$. Notice that $K_{i}$ is a positive definite Toeplitz kernel. Applying Theorem 3.5, the result follows. 


\section{Isometric dilations of contractive representations of some semigroups}

Let $G$ be a discrete additive subgroup of the real numbers and $G^{+}$be the unital semigroup of nonnegative elements of $G$. Define the free product semigroup

$$
{ }_{n} G^{+}:=G_{1}^{+} * \cdots * G_{n}^{+},
$$

where $G_{i}^{+}$is an isomorphic copy of $G^{+}$for any $i=1,2, \ldots, n$. A reduced word in $*_{n} G^{+}$has the form

$$
\sigma=t_{i_{1}} * t_{i_{2}} * \cdots * t_{i_{k}},
$$

where $\quad i_{1} \neq i_{2} \neq \cdots \neq i_{k}, i_{1}, \ldots, i_{k} \in\{1,2, \ldots, n\}, \quad$ and $\quad t_{i_{j}} \in G_{i_{j}}^{+} \backslash\{0\}$ $(j=1,2, \ldots, k)$.

Let $\left\{T_{i}(s)\right\}_{s \in G^{+}} \subset B(\mathscr{H})(i=1,2, \ldots, n)$ be semigroups of operators, i.e.,

$$
T_{i}(0)=I_{\mathscr{H}}, \quad T_{i}(s+t)=T_{i}(s) T_{i}(t)
$$

for any $s, t \in G^{+}, i=1,2, \ldots, n$, and such that

$$
T_{1}(s) T_{1}(s)^{*}+\cdots+T_{n}(s) T_{n}(s)^{*} \leq I_{\mathscr{H}}
$$

for any $s \in G^{+} \backslash\{0\}$. A sequence of semigroups of operators satisfying (4.1) is called contractive. For such $G^{+}$, there always exist semigroups of operators which have the desired properties (see Section 5).

For any $\sigma=t_{i_{1}} * t_{i_{2}} * \cdots * t_{i_{k}} \in *_{n} G^{+}$with $i_{1} \neq i_{2} \neq \cdots \neq i_{k}$, let us define

$$
T(\sigma):=T_{i_{1}}\left(t_{i_{1}}\right) \cdots T_{i_{k}}\left(t_{i_{k}}\right) .
$$

Notice that $T(\sigma) T(\omega)=T(\sigma \omega)$ for any $\sigma, \omega \in *_{n} G^{+}$, thus $\{T(\sigma)\}_{\sigma \in *_{n} G^{+}}$is a semigroup of contractions.

Assume that $G^{+}$is commensurable, i.e., it has the property that for any $t_{1}, t_{2}, \ldots, t_{k} \in G^{+} \backslash\{0\}$ there is $t \in G^{+}$and $n_{1}, \ldots, n_{k} \in\{1,2, \ldots\}$ such that $t_{i}=n_{i} t$ for any $i=1,2, \ldots, k$. For instance $G^{+}=\mathrm{Z}^{+}, G^{+}=\mathrm{Q}^{+}$, etc.

Let us define the kernel $K:\left(*_{n} G^{+}\right) \times\left(*_{n} G^{+}\right) \rightarrow B(\mathscr{H})$ by $K(0,0)=I_{\mathscr{H}}$ and

$$
K(\sigma, \omega)= \begin{cases}K(\tau, 0) & \text { if } \sigma=\omega \tau \text { for some } \tau \in *_{n} G^{+} \\ K(0, \tau) ; & \text { if } \omega=\sigma \tau \text { for some } \tau \in *_{n} G^{+} \\ 0 ; & \text { otherwise }\end{cases}
$$

where $K(0, \tau)=T(\tau)=K(\tau, 0)^{*}$. We shall prove that

$$
\sum_{i, j=1}^{k}\left\langle K\left(\sigma_{i}, \sigma_{j}\right) h_{j}, h_{i}\right\rangle \geq 0
$$


for any $\sigma_{1}, \sigma_{2}, \ldots, \sigma_{k} \in *_{n} G^{+}$and $h_{1}, \ldots, h_{k} \in \mathscr{H}$. Assume that $\sigma=$ $t_{i_{1}} * t_{i_{2}} * \cdots * t_{i_{k}}$ with $t_{i_{j}} \in G_{i_{j}}^{+}=G^{+}, t_{i_{j}} \neq 0$. Then there exist $t \in G^{+} \backslash\{0\}$ and $n_{1}, \ldots, n_{k} \in\{1,2, \ldots\}$ such that $t_{i_{j}}=n_{j} t$. Therefore,

$$
T(\sigma)=T_{i_{1}}(t)^{n_{1}} \cdots T_{i_{k}}(t)^{n_{k}}=T(t)(\epsilon),
$$

where $\epsilon=s_{i_{1}}^{n_{1}} \cdots s_{i_{k}}^{n_{k}} \in \mathrm{F}_{n}^{+}$(see Example 2.7). If $\sigma_{1}, \sigma_{2}, \ldots, \sigma_{k} \in *_{n} G^{+}$, then a similar argument shows that there exists $t \in G^{+}$and $\epsilon_{1}, \epsilon_{2}, \ldots, \epsilon_{k} \in \mathrm{F}_{n}^{+}$such that

$$
K\left(\sigma_{i}, \sigma_{j}\right)=K_{\left[T_{1}(t), \ldots, T_{n}(t)\right]}\left(\epsilon_{i}, \epsilon_{j}\right) .
$$

Since

$$
T_{1}(t) T_{1}(t)^{*}+\cdots+T_{n}(t) T_{n}(t)^{*} \leq I_{\mathscr{H}},
$$

we infer, according to Example 2.7, that

$$
\sum_{i=1}^{k}\left\langle K_{\left[T_{1}(t), \ldots, T_{n}(t)\right]}\left(\epsilon_{i}, \epsilon_{j}\right) h_{j}, h_{i}\right\rangle \geq 0 .
$$

Therefore, the relation (4.3) holds for any $\sigma_{1}, \ldots, \sigma_{k} \in *_{n} G^{+}$and $h_{1}, \ldots, h_{k} \in$ $\mathscr{H}$. Hence, $K$ is a positive definite Toeplitz kernel.

Let $G$ be a discrete additive subgroup of the real numbers such that $G^{+}$is commensurable.

THEOREM 4.1. Let $\left\{T_{i}(s)\right\}_{s \in G^{+}} \subset B(\mathscr{H}) \quad(i=1,2, \ldots, n)$ be a contractive sequence of semigroups of operators. Then there exists a Hilbert space $\mathscr{K} \supset \mathscr{H}$ and $\left\{V_{i}(s)\right\}_{s \in G^{+}} \subset B(\mathscr{K}) \quad(i=1,2, \ldots, n)$ a contractive sequence of semigroups of isometries such that

$$
T_{i_{1}}\left(s_{1}\right) T_{i_{2}}\left(s_{2}\right) \cdots T_{i_{k}}\left(s_{k}\right)=\left.P_{\mathscr{H}} V_{i_{1}}\left(s_{1}\right) V_{i_{2}}\left(s_{2}\right) \cdots V_{i_{k}}\left(s_{k}\right)\right|_{\mathscr{H}}
$$

for any $k \in\{1,2, \ldots\}, i_{1}, \ldots, i_{k} \in\{1,2, \ldots, n\}, s_{1}, s_{2}, \ldots, s_{k} \in G^{+}$, and

$$
\mathscr{K}=\bigvee V_{i_{1}}\left(s_{1}\right) \cdots V_{i_{k}}\left(s_{k}\right) \mathscr{H}
$$

where the span is taken over all $i_{1}, \ldots, i_{k} \in\{1, \ldots, n\}, s_{1}, \ldots, s_{k} \in G^{+}$, and $k=1,2, \ldots$.

Moreover, the isometric dilation satisfying these properties is uniquely determined up to an isomorphism.

Proof. Theorem 3.2 shows that the positive definite Toeplitz kernel defined by (4.2) has a Naimark dilation, i.e., there is a Hilbert space $\mathscr{K} \supset \mathscr{H}$ and $\{V(\sigma)\}_{\sigma \in *_{n} G^{+}}$a semigroup of isometries on $\mathscr{K}$ such that

$$
K(\sigma, \omega)=\left.P_{\mathscr{H}} V(\sigma)^{*} V(\omega)\right|_{\mathscr{H}}
$$


for any $\sigma, \omega \in P$ and $\mathscr{K}=\bigvee_{\sigma \in *_{n} G^{+}} V_{\sigma} \mathscr{H}$. Notice that if $t_{i} \in G_{i}^{+} \backslash\{0\}$, $t_{j} \in G_{j}^{+} \backslash\{0\}(i \neq j)$, then

$$
K\left(t_{i} * \sigma, t_{j} * \omega\right)=0 \text { for any } \sigma, \omega \in *_{n} G^{+} .
$$

According to Remark 3.3 part (iii), $V\left(t_{i}\right) \mathscr{K} \perp V\left(t_{j}\right) \mathscr{K}$. Setting $V_{i}(t):=$ $V\left(t_{i}\right)$, where $i=1,2, \ldots, n$, and $t_{i}=t \in G^{+}$, it follows that $\left\{V_{i}(t)\right\}_{t \in G^{+}}$ $(i=1,2, \ldots, k)$ are semigroups of isometries with

$$
V_{1}(t) V_{1}(t)^{*}+\cdots+V_{n}(t) V_{n}(t)^{*} \leq I_{\mathscr{K}}
$$

for any $t \in G^{+} \backslash\{0\}$. It is easy to see that the relations (4.4) and (4.5) are satisfied. The proof is complete.

Remark 4.2. Using Theorem 3.5, the result of Theorem 4.1 can be extended to systems of contractive sequences of semigroups of operators.

\section{Universal algebras associated to some semigroups of operators}

Let $G$ be a discrete additive subgroup of the real numbers and let $G^{+}$be the unital semigoup of nonnegative elements of $G$. Define the free product semigroup $*_{n} G^{+}:=G_{1}^{+} * \cdots * G_{n}^{+}$, where $G_{i}^{+}$is an isomorphic copy of $G^{+}$, for any $i=1,2, \ldots, n$. A reduced word in $*_{n} G^{+}$has the form

$$
\sigma=t_{i_{1}} * t_{i_{2}} * \cdots * t_{i_{k}},
$$

where $\quad i_{1} \neq i_{2} \neq \cdots \neq i_{k}, i_{1}, \ldots, i_{k} \in\{1,2, \ldots, n\}, \quad$ and $\quad t_{i_{j}} \in G_{i_{j}}^{+} \backslash\{0\}$ $(j=1,2, \ldots, k)$.

The length of $\sigma$ is defined to be $|\sigma|:=t_{i_{1}}+\cdots+t_{i_{k}}$. It is clear that $*_{n} G^{+}$is a unital semigroup with the left cancellation property and no divisors of the neutral element $0 \in *_{n} G^{+}$. Let

$$
\ell^{2}\left(*_{n} G^{+}\right)=\left\{f:\left.*_{n} G^{+} \rightarrow \mathrm{C}\left|\sum_{\sigma \in *_{n} G^{+}}\right| f(\sigma)\right|^{2}<\infty\right\}
$$

and let $\left\{\delta_{\sigma}\right\}_{\sigma \in *_{n} G^{+}}$be its canonical basis, i.e., $\delta_{\sigma}(\omega)=1$ if $\omega=\sigma$ and $\delta_{\sigma}(\omega)=0$ otherwise. For each $i=1,2, \ldots, n$ and $t \in G^{+}$, define the operator $S_{i}(t)$ on the Hilbert space $\ell^{2}\left(*_{n} G_{+}\right)$by

$$
S_{i}(t)\left(\sum_{\tau \in *_{n} G^{+}} \delta_{\tau} \lambda_{\tau}\right)=\sum_{\tau \in *_{n} G^{+}} \delta_{t^{(i)} * \tau} \lambda_{\tau}, \quad t=t^{(i)} \in G_{i}^{+},
$$

where $\lambda_{\tau} \in \mathrm{C}$ and $\sum_{\tau \in *_{n} G^{+}}\left|\lambda_{\tau}\right|^{2}<\infty$.

Since the semigroup $*_{n} G^{+}$has the left cancellation property and no divi- 
sors of the identity, it is easy to see that for any $i=1,2, \ldots, n$, and $t \in G^{+} \backslash\{0\}$, the operator $S_{i}(t)$ is a unilateral shift on $\ell^{2}\left(*_{n} G^{+}\right)$.

For any reduced word $\sigma=t_{i_{1}} * t_{i_{2}} * \cdots * t_{i_{k}}$ define $S(\sigma):=$ $S_{i_{1}}\left(t_{i_{1}}\right) \cdots S_{i_{k}}\left(t_{i_{k}}\right)$. Thus, $\{S(\sigma)\}_{\sigma \in *_{n} G^{+}}$is an isometric representation of the semigroup $*_{n} G^{+}$on the Hilbert space $\ell^{2}\left(*_{n} G^{+}\right)$. Notice that

$$
\ell^{2}\left(*_{n} G^{+}\right)=\bigoplus_{\sigma \in *_{n} G^{+}} S(\sigma)(\mathrm{C}) .
$$

Using (5.1), one can see that

$$
S_{i}(0)=I, \quad S_{i}\left(t_{1}\right) S_{i}\left(t_{2}\right)=S_{i}\left(t_{1}+t_{2}\right)
$$

for any $i=1,2, \ldots, n$, and $t_{1}, t_{2} \in G^{+}$. Thus $\left\{S_{i}(t)\right\}_{t \in G^{+} \backslash\{0\}}$ is a semigroup of unilateral shifts. On the other hand, if $i \neq j, i, j \in\{1,2, \ldots, n\}$ then $S_{j}^{*}(t) S_{i}(s)=0$ for any $t, s \in G^{+} \backslash\{0\}$. Therefore,

$$
S_{1}(t) S_{1}(t)^{*}+\cdots+S_{n}(t) S_{n}(t)^{*}<I_{\ell^{2}\left({ }_{n} G^{+}\right)}
$$

for any $t \in G^{+} \backslash\{0\}$.

For each $n=1,2, \ldots$, denote by $\mathscr{A}_{n}\left(G^{+}\right)$the non-selfadjoint closed algebra generated by $\left\{S_{i}(t)\right\}_{i=1,2, \ldots, n} \in G_{+}$and let $C_{r}^{*}\left(*_{n} G^{+}\right)$be the $C^{*}$-algebra generated by $\left\{S_{i}(t)\right\}_{\substack{t \in G_{+} \\ i=1, \ldots, n}}$. Notice that in the particular case when $G^{+}=\mathbf{Z}^{+}=\{0,1,2, \ldots\}, \mathscr{A}_{n}\left(\mathbf{Z}^{+}\right)$is the noncommutative disc algebra $\mathscr{A}_{n}$ introduced in [Pol], and $C_{r}^{*}\left(*_{n} \mathbf{Z}^{+}\right)=C^{*}\left(S_{1}, \ldots, S_{n}\right)$ is the extension through compacts of the Cuntz algebra $\mathcal{O}_{n}(n \geq 2)$.

Let $\left\{T_{i}(s)\right\}_{s \in G^{+}} \subset B(\mathscr{H})(i=1,2, \ldots, n)$ be semigroups of operators, i.e.,

$$
T_{i}(0)=I, \quad T_{i}(s+t)=T_{i}(s) T_{i}(t)
$$

for any $s, t \in G^{+}, i=1,2, \ldots, n$, and such that

$$
T_{1}(s) T_{1}(s)^{*}+\cdots+T_{n}(s) T_{n}(s)^{*} \leq I_{\mathscr{H}}
$$

for any $s \in G^{+} \backslash\{0\}$.

For such $G^{+}$, there always exist semigroups of contractions which have the desired properties. If $\mathscr{K}$ is a Hilbert space, then $\left\{S(\sigma) \otimes I_{\mathscr{K}}\right\}_{\sigma \in *_{n} G^{+}}$is the left regular representation of $*_{n} G^{+}$on $\ell^{2}\left(*_{n} G_{+}\right) \otimes \mathscr{K}$. For any $s_{0}>0$, define

$$
\mathscr{K}_{s_{0}}=\bigoplus_{\substack{\sigma \in *_{n} G^{+} \\|\sigma| \leq s_{0}}}\left(S(\sigma) \otimes I_{\mathscr{K}}\right)(\mathscr{K}) .
$$

Notice that $S(\omega)^{*}\left(\mathscr{K}_{s_{0}}\right) \subset \mathscr{K}_{s_{0}}$ for any $\omega \in *_{n} G^{+}$. Define $T_{i}(t)=$ $\left.P_{\mathscr{K}_{s_{0}}} S_{i}(t)\right|_{\mathscr{K}_{s_{0}}}$, for any $i=1,2, \ldots, n$ and $t \in G^{+}$. It is easy to see that 
$\left\{T_{i}(t)\right\}_{t \in G^{+}} \subset B\left(\mathscr{K}_{s_{0}}\right)(i=1,2, \ldots, n)$ satisfies the above-mentioned properties.

We need now a few definitions. We identify $M_{m}(B(\mathscr{H}))$, the set of $m \times m$ matrices with entries from $B(\mathscr{H})$, with $B(\underbrace{\mathscr{H} \oplus \cdots \oplus \mathscr{H}}_{\mathrm{m} \text {-times }})$. Thus we have a natural $C^{*}$-norm on $M_{m}(B(\mathscr{H}))$. If $X$ is an operator space, i.e., a linear subspace of $B(\mathscr{H})$, we consider $M_{m}(X)$ as a subspace of $M_{m}(B(\mathscr{H}))$ with the induced norm. The appropriate morphisms between operator spaces are the completely bounded maps [Ar1], [P2], [Pi]. Let $X, Y$ be operator spaces and $u: X \rightarrow Y$ be a linear map. Define $u_{m}: M_{m}(X) \rightarrow M_{m}(Y)$ by

$$
u_{m}\left(\left[x_{i j}\right]\right)=\left[u\left(x_{i j}\right)\right] .
$$

We say that $u$ is completely bounded (cb in short) if

$$
\|u\|_{\mathrm{cb}}=\sup _{m \geq 1}\left\|u_{m}\right\|<\infty .
$$

If $\|u\|_{\mathrm{cb}} \leq 1$ (resp. $u_{m}$ is an isometry for any $m \geq 1$ ) then $u$ is completely contractive (resp. isometric), and if $u_{m}$ is positive for all $m$, then $u$ is called completely positive.

From now on we assume that $G^{+}$is a commensurable unital subsemigroup of $[0, \infty)$.

TheOREM 5.1. Let $\left\{A_{i}(t)\right\}_{t \in G^{+}} \subset B(\mathscr{H}), i=1,2, \ldots, n$, be $n$ semigroups of operators. Then

$$
A_{1}(t) A_{1}(t)^{*}+\cdots+A_{n}(t) A_{n}(t)^{*} \leq I_{\mathscr{H}} \quad \text { for any } t \in G^{+} \backslash\{0\}
$$

if and only if the linear map $\Phi: \mathscr{A}_{n}\left(G^{+}\right) \rightarrow B(\mathscr{H})$ defined by

$$
\Phi\left(S_{i_{1}}\left(t_{1}\right) \cdots S_{i_{k}}\left(t_{k}\right)\right)=A_{i_{1}}\left(t_{1}\right) \cdots A_{i_{k}}\left(t_{k}\right)
$$

for any $i_{1}, \ldots, i_{k} \in\{1,2, \ldots, n\}, t_{1}, \ldots, t_{k} \in G^{+}$and $k \in\{1,2, \ldots\}$ is a completely contractive homomorphism.

Proof. If $G^{+}$is not dense the result can be deduced from [Po2, Theorem 2.1]. Assume that $G^{+}$is dense in $[0, \infty)$ and the relation (5.5) holds. According to Theorem 4.1, there exists a Hilbert space $\mathscr{K} \supset \mathscr{H}$ and $\left\{V_{i}(t)\right\}_{t \in G^{+}} \quad(i=1,2, \ldots, n)$ semigroups of isometries such that

$$
V_{1}(t) V_{1}(t)^{*}+\cdots+V_{n}(t) V_{n}(t)^{*} \leq I_{\mathscr{H}}, \quad t \in G^{+} \backslash\{0\}
$$

and

$$
A_{i_{1}}\left(s_{1}\right) \cdots A_{i_{k}}\left(s_{k}\right)=\left.P_{\mathscr{H}} V_{i_{1}}\left(s_{1}\right) \cdots V_{i_{k}}\left(s_{k}\right)\right|_{\mathscr{H}}
$$


for any $i_{1}, \ldots, i_{k} \in\{1,2, \ldots, n\}, s_{1}, \ldots, s_{k} \in G^{+}$, and $k \in\{1,2, \ldots\}$. According to [D2], since $G^{+}$is dense, there is a $*$-isomorphism

$$
\Psi: C^{*}\left(S_{1}(t), \ldots, S_{n}(t)\right) \rightarrow C^{*}\left(V_{1}(t), \ldots, V_{n}(t)\right)
$$

such that

$$
\Psi\left(S_{i_{1}}\left(t_{1}\right) \cdots S_{i_{k}}\left(t_{k}\right) S_{j_{1}}\left(s_{1}\right)^{*} \cdots S_{j_{p}}\left(s_{p}\right)^{*}\right)=V_{i_{1}}\left(t_{1}\right) \cdots V_{i_{k}}\left(t_{k}\right) V_{j_{1}}\left(s_{1}\right)^{*} \cdots V_{j_{p}}\left(s_{p}\right)^{*} .
$$

In particular, we have $\|\Psi\|_{\mathrm{cb}}=1$. According to (5.7), we have

$$
\begin{aligned}
\Phi\left(S_{i_{1}}\left(t_{1}\right) \cdots S_{i_{k}}\left(t_{k}\right)\right) & =A_{i_{1}}\left(t_{1}\right) \cdots A_{i_{k}}\left(t_{k}\right) \\
& =\left.P_{\mathscr{H}} V_{i_{1}}\left(t_{1}\right) \cdots V_{i_{k}}\left(t_{k}\right)\right|_{\mathscr{H}} \\
& =\left.P_{\mathscr{H}} \Psi\left(S_{i_{1}}\left(t_{1}\right) \cdots S_{i_{k}}\left(t_{k}\right)\right)\right|_{\mathscr{H}} .
\end{aligned}
$$

Similarly, one can show that

$$
\Phi(f)=\left.P_{\mathscr{H}} \Psi(f)\right|_{\mathscr{H}} \quad \text { for any } f \in \mathscr{A}_{n}\left(G^{+}\right) .
$$

Therefore, $\|\Phi\|_{\mathrm{cb}} \leq\|\Psi\|_{\mathrm{cb}}=1$.

Conversely, assume that $\left\{A_{i}(t)\right\}_{t \in G^{+}} \subset B(\mathscr{H}), i=1,2, \ldots n$, are semigroups of operators such that the linear map $\Phi$, defined by (5.6), is completely contractive. In particular, we have

$$
\left\|\left[\begin{array}{cccc}
A_{1}(t) & A_{2}(t) & \cdots & A_{n}(t) \\
0 & 0 & \cdots & 0 \\
\vdots & \vdots & & \vdots \\
0 & 0 & \cdots & 0
\end{array}\right]\right\| \leq\left\|\left[\begin{array}{cccc}
S_{1}(t) & S_{2}(t) & \cdots & S_{n}(t) \\
0 & 0 & \cdots & 0 \\
\vdots & \vdots & & \vdots \\
0 & 0 & \cdots & 0
\end{array}\right]\right\|,
$$

which shows that

$$
A_{1}(t) A_{1}(t)^{*}+\cdots+A_{n}(t) A_{n}(t)^{*} \leq I_{\mathscr{H}} \quad \text { for any } t \in G^{+} \backslash\{0\} .
$$

The proof is complete.

Using Paulsen's result [P1] and Theorem 5.1, one can infer the following.

COROllary 5.2. A representation $\Phi: \mathscr{A}_{n}\left(G^{+}\right) \rightarrow B(\mathscr{H})$ is completely bounded if and only if it is given by $\Phi\left(S_{i}(t)\right)=X T_{i}(t) X^{-1}$, $i=1,2, \ldots, n, t \in G^{+}$, where $\left\{T_{i}(t)\right\}_{t \in G^{+}}(i=1,2, \ldots, n)$ are semigroups of operators with

$$
T_{1}(t) T_{1}(t)^{*}+\cdots+T_{n}(t) T_{n}(t)^{*} \leq I_{\mathscr{H}} \quad \text { for any } t \in G^{+} \backslash\{0\},
$$

and $X$ is an invertible operator.

Let $G$ be a discrete additive subgroup of the real numbers and define the free 
product group $*_{n} G=G_{1} * \cdots * G_{n}$, where $G_{i}$ is an isomorphic copy of $G$ for any $i=1,2, \ldots, n$. Let

$$
\ell^{2}\left(*_{n} G\right)=\left\{f: *_{n} G \rightarrow \mathrm{C} \sum_{\sigma \in *_{n} G}|f(\sigma)|^{2}<\infty\right\}
$$

and let $\left\{e_{\sigma}\right\}_{\sigma \in *_{n} G}$ be the canonical basis of $\ell^{2}\left(*_{n} G\right)$, i.e., $e_{\sigma}(t)=1$ if $t=\sigma$ and $e_{\sigma}(t)=0$ otherwise. For each $i=1,2, \ldots, n$ and $t \in G$, let $U_{j}(t) \in B\left(\ell^{2}\left(*_{n} G\right)\right)$ be the unitary operator defined by

$$
U_{i}(t)\left(\sum_{\sigma \in *_{n} G} \lambda_{\sigma} e_{\sigma}\right)=\sum_{\sigma \in *_{n} G} \lambda_{\sigma} e_{t^{(i)} * \sigma} \quad\left(t=t^{(i)} \in G_{i}\right),
$$

where $\lambda_{\sigma} \in \mathrm{C}$ and $\sum_{\sigma \in *_{n} G}\left|\lambda_{\sigma}\right|^{2}<\infty$. It is clear that $\left\{U_{i}(t)\right\}_{t \in G^{+}}$ $(i=1,2, \ldots, n)$ are semigroups of unitary operators. The Hilbert space $\ell^{2}\left(*_{n} G^{+}\right)$can be seen as a subspace of $\ell^{2}\left(*_{n} G\right)$ and

$$
\left.U_{i}(t)\right|_{\ell^{2}\left(*_{n} G^{+}\right)}=S_{i}(t)
$$

for any $t \in G^{+}, i=1,2, \ldots, n$.

Now, one can prove the following unitary dilation theorem for contractive semigroups of operators. Assume that $G^{+}$is commensurable.

Theorem 5.3. Let $\left\{T_{i}(t)\right\}_{t \in G^{+}} \subset B(\mathscr{H}), i=1,2, \ldots, n$, be a sequence of semigroups of operators such that

$$
T_{1}(t) T_{1}(t)^{*}+\cdots+T_{n}(t) T_{n}(t)^{*} \leq I_{\mathscr{H}}, \text { for any } t \in G^{+} \backslash\{0\} .
$$

Then there is a Hilbert space $\mathscr{K} \supset \mathscr{H}$ and a sequence $\left\{W_{i}(t)\right\}_{t \in G^{+}} \subset B(\mathscr{K})$, $i=1,2, \ldots, n$, of unitary semigroups such that $\mathscr{K}$ is the smallest closed reducing subspace for each $W_{i}(t)\left(t \in G^{+}, i=1,2, \ldots, n\right)$, containing $\mathscr{H}$, and such that

$$
T_{i_{1}}\left(t_{1}\right) \cdots T_{i_{k}}\left(t_{k}\right)=\left.P_{\mathscr{H}} W_{i_{1}}\left(t_{1}\right) \cdots W_{i_{k}}\left(t_{k}\right)\right|_{\mathscr{H}}
$$

for any $i_{1}, \ldots, i_{k} \in\{1,2, \ldots, n\}$ and $t_{1}, \ldots, t_{k} \in G^{+}$.

Proof. Let $\mathscr{P}_{\mathscr{U}}$ be the set of all polynomials in $U_{i}(t), t \in G^{+}, i=1, \ldots, n$. According to the Theorem 5.1 and the relation (5.8), the linear map $\mathscr{X}: \mathscr{P}_{\mathscr{U}} \rightarrow B(\mathscr{H})$ defined by

$$
\mathscr{X}\left(U_{i_{1}}\left(t_{1}\right) \cdots U_{i_{k}}\left(t_{k}\right)\right)=T_{i_{1}}\left(t_{1}\right) \cdots T_{i_{k}}\left(t_{k}\right)
$$

for any $i_{1}, \ldots, i_{k} \in\{1,2, \ldots, n\}$ and $t_{1}, \ldots, t_{k} \in G^{+}$, is a completely contractive homomorphism. Applying Arverson's extension theorem [Ar1, Theorem 1.2.9] to our setting, there is a completely positive linear map 


$$
\tilde{X}: C^{*}\left(\left\{U_{i}(t)\right\}\right) \rightarrow B(\mathscr{H})
$$

such that $\left.\tilde{\mathscr{X}}\right|_{\mathscr{P}_{\text {ol }}}=\mathscr{X}$. Combining this result with Stinespring's representation $[\mathrm{S}]$ the result follows.

According to the notation from Section 1, we set

$$
p\left(\left\{T_{i}(t)\right\}\right):=\tilde{\rho}_{\tau}(p) \text { for any } p \in \mathscr{P}\left(*_{n} G^{+}\right) .
$$

Using Theorem 5.1 and Theorem 5.3, we infer the following version of the von Neumann inequality [vN], which also extends the main result from [Po1] to contractive semigroups of operators.

Corollary 5.4. Let $\left\{T_{i}(t)\right\}_{t \in G^{+}} \subset B(\mathscr{H}), i=1,2, \ldots, n$, be a sequence of semigroups of operators such that

$$
T_{1}(t) T_{1}(t)^{*}+\cdots+T_{n}(t) T_{n}(t)^{*} \leq I_{\mathscr{H}}, \quad t \in G^{+} \backslash\{0\} .
$$

Then

$$
\left\|p\left(\left\{T_{i}(t)\right\}\right)\right\| \leq\|p\|_{C_{r}^{*}\left(*_{n} G^{+}\right)} \leq\|p\|_{C^{*}\left(*_{n} G\right)}
$$

for any polynomial $p \in \mathscr{P}\left(*_{n} G^{+}\right)$, which is viewed as an element of $C_{r}^{*}\left(*_{n} G^{+}\right)\left(\operatorname{resp} . C^{*}\left(*_{n} G\right)\right)$.

It is plain that the inequality (5.9) can be extended to matrices $M_{m}\left(\mathscr{P}\left(*_{n} G^{+}\right)\right)$.

\section{REFERENCES}

[Ar1] W. B. Arveson. Subalgebras of $C^{*}$-algebras, Acta. Math. 123 (1969), 141-224 .

[Ar2] W. B. Arveson, Continuous analogues of Fock space, Mem. Amer. Math. Soc. 80 (1989), No. 409.

[B] M. Bozejko, Positive definite kernels, length functions on groups and noncommutative von Neumann inequality, Studia Math. 95 (1989), 107-118.

[C1] L .A. Coburn, The $C^{*}$-algebra generated by an isometry, I, Bull. Amer. Math .Soc. 13 (1967), 722-726.

[C2] L.A. Coburn, The $C^{*}$-algebra generated by an isometry, II, Trans. Amer. Math. Soc. 137 (1969), 211-217.

[Cu] J. Cuntz, Simple $C^{*}$-algebras generated by isometries, Commun. Math. Phys. 57 (1977), 73-185.

[D1] H. T. Dinh, Discrete product systems and their $C^{*}$-algebras, J. Funct. Anal. 102 (1991), $1-34$.

[D2] H. T. Dinh, On generalized Cuntz $C^{*}$-algebras, J. Operator Theory 30 (1993), 123-135.

[Do] R. G. Douglas, On the $C^{*}$-algebra of one-parameter semigroup of isometries, Acta Math. 128 (1972), 143-152.

[N] M. A. Naimark, Positive definite operator functions on a commutative group, Bulletin Acad. Sci. URSS 7 (1943), 237-244.

[P1] V. I. Paulsen, Every completely polynomially bounded operator is similar to a contraction, J. Funct. Anal. 55 (1984), 1-17. 
[P2] V. I. Paulsen, Completely Bounded Maps and Dilations, Pitman Research Notes in Mathematics, Vol. 146, New York, 1986.

[Pi] G. Pisier, Similarity Problems and Completely Bounded Maps, Lecture Notes in Math. 1618 (1995).

[Po1] G. Popescu, Von Neumann inequality for $\left(B(H)^{n}\right)_{1}$ Math. Scand. 68 (1991), 292-304.

[Po2] G. Popescu, Non-commutative disc algebras and their representations, Proc. Amer. Math. Soc. 124 (1996), 2137-2148.

[Po3] G. Popescu, Positive-definite functions on free semigroups, Canad. J. Math. 48 (1996), 887-896.

[Po4] G. Popescu, Noncommutative joint dilations and free product operator algebras, Pacific J. Math. 186 (1998), 111-140.

[S] W. F. Stinespring, Positive functions on $C^{*}$-algebras, Proc. Amer. Math. Soc. 6 (1955).

[SzF] B. Sz.-Nagy, C. Foias Harmonic Analysis on Operators on Hilbert Space 1970 NorthHolland, Amsterdam (1970).

[vN] J. von Neumann, Eine Spectraltheorie für allgemeine Operatoren eines unitären Raumes, Math. Nachr.4 (1951), 258-281.

DIVISION OF MATHEMATICS AND STATISTICS

THE UNIVERSITY OF TEXAS AT SAN ANTONIO, SAN ANTONIO TX 78249

U.S.A.

Email: gpopescu@sphere.math.utsa.edu 